\title{
Carbogenic Molecular Sieves for Reaction and Separation by Design: A Novel Approach to Shape Selective Super Base, Super Acid and Catalytic Membranes.
}

DOE-BES Contract No. DE-FG02-97ER 14802

FINAL REPORT

September 1997 - November 2000 plus 1 year no cost extension

Professor Henry C. Foley

Department of Chemical Engineering

University of Delaware

\author{
currently at \\ Department of Chemical Engineering \\ The Pennsylvania State University \\ University Park, PA 16801 \\ hcf2@psu.edu \\ 814-865-2574 PH \\ 814-865-7846 FAX
}

DOE Patent Clearance Granted

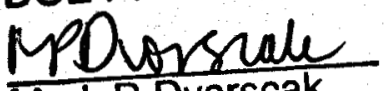

Mark P. Dvorscak

(630) 252-2393

(1) doe.gov

E-mail: mark. Intectual Property Law

Office of Intellectual Propertions Office

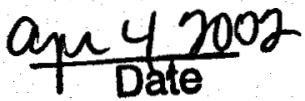




\section{DISCLAMMER}

This report was prepared as an account of work sponsored by an agency of the United States Government. Neither the United States Governmeat nor any agency thereof, aor any of their employees, makes any warranty, express or implied, of assumes any legal liability or responsibility for the accuracy, completeness, or usefulaess of any information, apparatus, product, or process disclosed, or represents that its use would not infringe privately owned rights. Reference berein to any specific commercial product, process, or service by trade name, trademark, manufacturex, or otherwise does not necessatily constitute or imply its endorsement, recommendation, or favoring by the Uaited States Government or any agency thereof. The views and opinions of authors expressed herein do not necessarily state or reflect those of the United States Government or any ageney thereof. 


\section{DISCLAIMER}

Portions of this document may be illegible in electronic image products. Images are produced from the best available original document. 


\section{Introduction}

This report summarizes the scientific results developed under the auspices of DOE-BES Contract Number DE-FG02-97ER14802, entitled "Carbogenic Molecular Sieves for Reaction and Separation by Design: A Novel Approach to Shape Selective Super Base, Super Acid and Catalytic Membranes," which was in place between VV/98 and MM/00 at the University of Delaware. This report and several of the papers which have been submitted during 2000 and 2001 were written by the principal investigator, Professor Foley, and his Ph.D. students, Dr. Michael S. Strano, Dr. Mark B. Shiflett, Dr. Madhav Acharya, Dr. Mark G. Stevens and Mr. Kevin McNamara. Drs. Strano, Acharya, Stevens and Shiflett received their $\mathrm{Ph} . \mathrm{D}$. degrees based upon research funded by this program. Dr. Shiflett, an employee of the Du Pont company and a Ph.D. student at the University of Delaware, was able to work full time on this work for approximately three years. In a very real way his time and salary at Du Pont were real in-kind support of the DOE-funded research. At the same time, many of the resources of the Du Pont Experimental Station were made available to me and to my other students during the course of the work. I am formally acknowledging the direct and indirect support of the Du Pont company in this research.

The proposed goals for this work were to design, fabricate and analyze supported nanoporous carbon membranes and to characterize and evaluate new catalysts comprised of bulk nanoporous carbons containing alkali metal introduced as the metal vapor or liquid for base catalyzed reactions.

The main findings of this three-year program of research are:

1. Excellent nanoporous carbon membranes with molecular sieving properties can be prepared on flat or tubular porous 316 stainless steel support media, providing stable, strong and mechanically robust asymmetric membranes.

2. The supported nanoporous carbon membranes (SNPCM) are highly selective for the separation of small molecules. SNPCM with ideal perm-selectivities for oxygen and nitrogen as high as 30:1 have been produced.

3. A model has been developed by which the ideal permselectivity ratio of oxygen to nitrogen is directly related to the fraction of the membrane pore area which is tied up in non-shape selective defect pores. Such defect pores when present as only $0.1 \%$ lead to $\mathrm{O}_{2} / \mathrm{N}_{2}$ permselectivity ratios of approximately $1: 1$, whereas when such pores make up just $0.0001 \%$ of the pore area, they ratio can be as high as 30:1. Values of this ratio between 30 and 1 indicate intermediate levels of defects. We have also shown that the $02 \mathrm{~N} 2$ test of permselectivity is an extremely stringent test of the membrane's quality, and that membranes with ratios as low as 6:1 show very high permselectivities with reaction even at reaction temperatures when larger molecules are involved. (see the case of MTBE cracking below.) 
4. Platinum metal nanoparticles (monodisperse and $\mathrm{rp}-3.5 \mathrm{~nm}$ ) may be synthesized spontaneously within the supported nanoporous carbon thin film during its preparation on the stainless steel support. The platinum nanoparticles have very high activity and the access to their surfaces is controlled by transport through the contiguous NPC solid. Hence the system shows molecular sieving coupled with catalytic reaction.

5. Heteropolyacid (12-tungstophosphoric acid, HPA) may be dispersed on the surface of the SNPCM with a resultant coupling of molecular sieving with catalytic reaction. When used to convert MTBE is cracked by the HPA to produce methanol and isobutylene. The best membrane continuously separates the methanol from the isobutene to the permeate side of the membrane, while the HPA catalyzes the conversion of the MTBE, which does not permeate. In this way the reaction may be taken to completion even at temperatures which would lead to less that $25 \%$ conversion at equilibrium. Significantly, the quality of the membrane as determined by the $02 / \mathrm{N} 2$ ideal permselectivity ratio is a good predictor of the outcome of the coupled catalysis and separation. If the ratio id $1: 1$, then the reaction the reactant and products permeate through the defect and sub-equilibrium conversions are obtained. If the ratio id 6:1 then only methanol permeates through the membrane and complete conversion and near perfect separation of the two products takes place, but total holding times must be very long. However, if the ratio is intermediate at 3:1, then the time holding time is an order of magnitude shorter and very little of the MTBE or isobutene slip to the permeate side with the methanol. Hence for catalytic reaction and separation with larger molecules, the membrane need not be as perfect as it would need to be for oxygen and nitrogen separation.

6. Cesium in nanoporous carbon ( $10-15 \mathrm{wt} \%)$ was observed to be an excellent catalyst for the heterogeneous conversion of 1-butene preferentially to cis-2butene, indicating an anionic mechanism of reaction. The same catalyst was also able to couple benzene to di- and triphenyl and to produce isobutylbenzene from propene and toluene under mile conditions. The catalyst will also convert acetone into isophorone and lesser amounts of mesityl oxide. It was also observed that when present at higher concentrations ( $>30 \mathrm{wt} \%)$, cesium catalyzes the formation of nanocrystalline domains from the amorphous nanoporous carbon. These domains are best described as multiwalled nanotubes, onions, polyhedra and nanographite. There formation is all the more unusual because the NPC is considered to be a non-crystallizing carbon, that is a carbon which converts to graphite only at temperatures approaching $2000^{\circ} \mathrm{C}$. In the presence of cesium, the nanocrystallization process takes place rapidly at temperatures as low as $350^{\circ} \mathrm{C}$.

\section{Synthesis, Characterization, and Application of Nanoporous Carbon Membranes}

Nanoporous carbon films have been synthesized on tubular porous metal supports ( $0.2 \mathrm{~m}$ porosity) for separating small molecules such as oxygen and nitrogen from air. The asymmetric carbon film is initially prepared by ultrasonically depositing a solution of 
polyfurfuryl alcohol and acetone on to a macroporous stainless steel (SS316L) tubular support (Shiflett and Foley, 1999). The coated support is rotated and pyrolyzed in a quartz tube furnace under an inert atmosphere at temperatures ranging from 600 to 900 $\mathrm{K}$. These supported nanoporous carbon membranes (SNPCM) show unprecedented size and shape selectivities. Using the rate of nitrogen $\left(\mathrm{N}_{2}\right)$ permeation for comparison, hydrogen $\left(\mathrm{H}_{2}\right)$, helium $(\mathrm{He})$, and oxygen $\left(\mathrm{O}_{2}\right)$ are transported through an SNPCM 330, 178,30 times faster. If the molecules were transported on the basis of Knudsen diffusion rather than on the basis of size selectivity, these transport ratios $\left(\mathrm{H}_{2} / \mathrm{N}_{2}, \mathrm{He} / \mathrm{N}_{2}\right.$, and $\mathrm{O}_{2} / \mathrm{N}_{2}$ ) would be $3.73,2.65$, and 0.94 , respectively.

Membrane synthesis and fabrication have proven to be problems with these materials, because formation of a continuous film, particularly over a large length scale, is difficult to achieve. A critical film thickness exists at about $22+/-1 \mathrm{~m}$ such that below this thickness defects in the carbon layer will reduce selectivity while exceeding this thickness leads to the detrimental formation of micro-cracks (Shiflett et al., 2000). Scanning electron microscopy (SEM) has been used extensively to characterize carbon film thickness, surface uniformity, and micro-crack formation. Digital enhancement of high resolution transmission electron micrographs (HRTEM) has confirmed "slit" shaped regions between graphitic planes which could reasonable represent nanopores. Small molecule selectivity can be explained by examining the pore size distribution calculated using methyl chloride adsorption data Mean pore size is centered round an average of 0.45 to $0.50 \mathrm{~nm}$ which is the size necessary to separate gases such as oxygen and nitrogen with molecular dimensions of 0.45 and $0.48 \mathrm{~nm}$, respectively.

Additives such as titanium dioxide, small pore high silica zeolites, and polyethylene glycol have been added to the polyfurfuryl alcohol precursor for modification of the carbon film to increase selectivity or flux (Shiflett and Foley, 2000). Altemative high energy heating sources such as a continuous carbon dioxide $\left(\mathrm{CO}_{2}\right)$ laser have also been investigated for reducing the time necessary to manufacture these thin supported carbon films.

In addition to transient experiments, steady-state separations were run to separate oxygen from air, remove nitrogen from natural gas, separate hydrogen from hydrogen cyanide, and separate fluorocarbons from hydrochloric acid. Permeate oxygen concentrations as high as 44 volume percent were achieved in continuous air separations -at room temperature. A $90 \%$ nitrogen removal from natural gas was accomplished at 196 $\mathrm{K}$. Selectivities of almost $100 \%$ were achieved removing hydrogen from hydrogen cyanide and fluorocarbons from hydrochloric acid.

Most of this experimental work was done with carbon membranes having outside surface area of about $5 \mathrm{~cm}^{2}$. Scale-up is necessary to produce modules for pilot scale separation studies. Reproducible carbon membrane fabrication with a larger outside surface area of $60 \mathrm{~cm}^{2}$ produced tubular membranes with fluxes within $+1-20 \%$ (Shiflett and Foley, 2001). Improved reproducibility is required along with higher fluxes before commercialization of this technology for gas-gas separations can be realized. A theoretical model which predicts the uniformity and thickness of a spray beam on a rotating and translating cylindrical support has been developed (Shiflett et al., 2001). Careful attention to the polymer deposition and pyrolysis processes should provide additional answers to improve reproducibility of high carbon membrane selectivities without sacrificing flux. 


\section{Reactive Nanoporous Membranes}

\section{Pt/Nanoporous Carbon Membranes}

A method for the synthesis of novel supported catalytic nanoporous carbon membranes was presented for use as a permselective catalytic membrane reactor (CMR). The method produces selective thin carbon films with the catalytic metal dispersed within the membrane layer. Scanning electron micrographs reveal that the membrane structure exists both within and external to the macroporosity of the stainless steel support. The effective thickness of these membranes from He permeation measurements is $\sim 12.5 \mu \mathrm{m}$. The Pt metal dispersion as estimated via TEM on membrane material was observed to have a volume averaged metal diameter of $7.1 \mathrm{~nm}$. Selective hydrogenation of monoolefins demonstrated the utility of the selective transport porosity of the membrane as well as the shape selective catalytic effects.

The catalytic membranes demonstrated moderate selectivity in the hydrogenation of olefins as measured as a ratio of product fluxes with 28.9:3.2:1 for propane: $n$-butane: isobutane at $125^{\circ} \mathrm{C}$. This was attributed primarily to moderately selective transport coefficients measured for the reactants and products from flux data. The ratios of observed hydrogenation rate constants measured for this system are larger than expected based on kinetic limitations alone: 62.8 and 5.3 for propylene/isobutylene and 1-butene/ isobutylene respectively at $125^{\circ} \mathrm{C}$. These results can be rationalized in terms of the failure of the hydrogenation model to account for the isotropic nature of the membrane material where reaction takes place in parallel to transport across the membrane. One consequence of this observation is that there exists considerable opportunity to improve the selectivities of these types of catalytic membranes as new synthesis approaches are developed leading to membranes with fewer defect pathways.

\section{Heteropolyacid Nanoporous Carbon Membranes}

A novel type of thin film catalyst has been developed that combines high activity with selective transport across a micron scale membrane film. Nanoporous carbon was used as a support for 12-tungstophosphoric acid with composite films synthesized by two distinct approaches: in-situ polymerization and adsorption from solution. Ion sputtering and XPS were used in combination to characterize the high dispersion of the catalyst within the film in the former case and a gradient in catalyst concentration in the latter. Both methods produce intact Keggin structures as demonstrated by FT-IR spectroscopy. Gas permeation experiments indicate a preservation of separation capacity with $\mathrm{O}_{2} / \mathrm{N}_{2}$ permeability ratios as high as 6.5 . The catalytic films were benchmarked using the decomposition of methyl tert-butyl ether at $55^{\circ} \mathrm{C}$ and demonstrate remarkable increases in both conversion and simultaneous separation of decomposition products. Integrated conversions in a semi-batch reactor configuration far exceed the thermodynamic limit of $67.4 \%$ at $55^{\circ} \mathrm{C}$ and $0.25 \mathrm{~atm}$ initial MTBE pressure to conversions approaching $99.9 \%$. At the same time, methanol and isobutylene are separated on either side of the membrane with methanol selectivities as high as $99.8 \%$ on the permeate side. These results indicate 
that this novel catalyst design and geometry have significant potential to increase reactor efficiency and simultaneously to reduce or eliminate subsequent separation processes in selected applications.

Nanoporous carbon membranes rendered catalytic in this way with $\mathrm{O}_{2} / \mathrm{N}_{2}$ ideal permselectivities greater than the Knudsen value can convert MTBE to products in yields well above equilibrium. As the $\mathrm{O}_{2} \mathrm{~N}_{2}$ permselectivity increases, the separation of isobutylene and methanol increases to near complete rejection of the MTBE and isobutylene but at the expense of membrane throughput. In contrast, membranes with Knudsen like selectivities do not adequately separate reactants and products and conversions are limited by thermodynamic equilibrium.

Heteropolyacid catalyst prepared within the nanoporous carbon is relatively inaccessible to MTBE and consequently, the effectiveness factor for the reaction is low. This low activity of these membranes is also consistent with the observation that MTBE transport within nanoporous carbon is hindered. Also, the acidic environment created during carbonization of the furfuryl alcohol/heteropolyacid mixture has been shown to lead to the development of viscous flow and Knudsen defect pathways and hence decreases nanopore surface area and membrane selectivity. Alternatively, catalyst adsorbed on the carbon surface of the membrane is highly accessible for reaction and is optimally placed.

For the first time, it has been shown that a nanoporous material can be used to drive reaction conversions to exceed thermodynamic equilibrium and simultaneously completely segregate products with relatively high purity. Hence, this next generation of catalytic composite materials yields enormous potential to revolutionize chemical processes by reducing downstream separations; lowering energy demands, increasing per pass conversions and minimizing mixed waste streams.

\section{Molecular Transport Across Nanoporous Membranes}

\section{Transient Analysis of Adsorption and Transport}

Transport through a supported nanoporous carbon membrane synthesized by ultrasonic deposition was studied as a function of temperature and pressure for a series of gas molecular probes: $\mathrm{O}_{2}, \mathrm{~N}_{2}, \mathrm{Ar}, \mathrm{He}, \mathrm{CO}_{2}$; and $\mathrm{SF}_{6}$. The permeation experiments were conducted at temperatures ranging from 25 to $225^{\circ} \mathrm{C}$ and over a range of pressures from 100 to $700 \mathrm{kPa}$. Permeances were weakly temperature dependent and did not necessarily exhibit activated transport as reported for similar membranes in the literature. Fluxes ranged from $10^{-7}$ to $10^{-4} \mathrm{~mol} / \mathrm{m}^{2} / \mathrm{s}$ with permeances ranging between $10^{-9}$ and $10^{-12} \mathrm{~mol} / \mathrm{m}^{2} / \mathrm{s} / \mathrm{Pa}$. Heats of adsorption were found to be $2.5,2.21,3.05$ and 2.52 $\mathrm{kcal} / \mathrm{mol}$ for $\mathrm{N}_{2}, \mathrm{O}_{2}, \mathrm{Ar}$ and $\mathrm{CO}_{2}$, respectively. These values are lower than those measured on granular nanoporous carbon. The apparent activation barriers to diffusion were also found to be quite low at $2.06,5.87,4.12,5.89$ and $2.19 \mathrm{kcal} / \mathrm{mol}$ for $\mathrm{He}, \mathrm{N}_{2}, \mathrm{O}_{2}$, Ar and $\mathrm{CO}_{2}$.

The results suggest the presence of two parallel pathways for transport - one through the nanopores and a second through larger defect pores. A model of permeation where transport occurs through nanoporous pathways in parallel with viscous flow defects was developed. The model describes some the non-linear phenomena observed in 
the measurement of energetic parameters, and shows that an area fraction of $50 \mathrm{~nm}$ defects as few as $3.43 \times 10^{-9}$ can suppress activation energies for permeation by 1 $\mathrm{kcal} / \mathrm{mol}$. Regressed parameter values from the model predict an $\mathrm{O}_{2} / \mathrm{N}_{2}$ selectivity of 10.7 (over the observed value of 4.3) which agrees well with this same value reported elsewhere in the literature for a similar carbon film.

\section{Parallel Transport Model of Membrane Permeation}

A parallel resistance transport model has been developed to describe the performance variation among carbon membranes appearing in the literature. The model considers permeation through the carbon film through high selectivity, high mass transport resistance nanopores in combination with flow through a small populations of larger, less selective defects. Although fewer in number, these larger pores exert a disproportionate influence on the total membrane flux because of their considerably lower mass transport resistance.

The model was applied to supported membranes synthesized by polymeric deposition and shown to describe the variations in permeability. A series of 200,50 and $1 \mathrm{~nm}$ average defect pore diameter was considered with the average pore size having little affect on the result model description. The predictive capabilities of the model were demonstrated using three membranes of increasing selectivity. Membranes with low gas separation ability (high numbers of defects) were shown to have a quadratic dependence of flux on pressure and this characteristic is well predicted by the model. Also, as membrane selectivity increases, the flux versus pressure response becomes linear as also predicted by the model. Using published data for polyimide based carbon membranes, the model was also shown to describe the $\mathrm{O}_{2} / \mathrm{N}_{2}$ selectivity variation with $\mathrm{O}_{2}$ permeability.

Also, activation energies for permeation are shown to be generally suppressed with increasing area fraction of defects which could account for the variability in reported activation energy values in the literature. Despite its simplicity and the complications involved in modeling vastly different synthesis methodologies, the model is shown to describe these variations well. Predictive capacity of the formalism is demonstrated in the description of the pressure dependence of permeation of membranes representing a range in selective performance.

\section{Mesoporous Adsorbent Synthesis by Templating of Pyrolytic Carbons}

\section{Synthesis of Ultrafiltration and Nanofiltration Membranes}

A novel method for producing carbon membranes for ultrafiltration applications has been developed using a spray deposition and pyrolysis of poly (furfuryl akchol)/poly (ethylene glycol) mixtures on macroporous stainless steel supports. The poly (ethylene glycol) or PEG employed as a carbonization template creates a mesoporosity that leads to pores in the ultrafiltration range. Scanning electron microscopy shows that the membranes consisted of $12-$ to $15-\mu \mathrm{m}$ thick carbon films. Gas permeation and water permeability data were used for the calculation of mean pore sizes, which were found to decrease with decreasing average molecular weight of the PEG template. Ultrafiltration 
of a polydisperse dextran solution was used to quantify the retention properties of the membranes. Molecular weight cutoffs determined from dextran retention data were shown to vary with template molecular weight: values of $2 \times 10^{4}, 3.5 \times 10^{4}$, and $6 \times 10^{4}$ $(\mathrm{g} / \mathrm{mol}$ dextran) were measured for respective templates of 2000,3400 , and $8000(\mathrm{~g} / \mathrm{mol}$ PEG). For PEG molecular weights of 2000 or below, the templating effect was ill defined, membrane film cracking became more prominent, and membrane selectivity and reproducibility were adversely affected.

\section{Compositional and Molecular Weight Dependence of Templating}

The process of templating porous carbons has been examined in greater detail by looking at the effect of molecular weight and composition of the template/carbonizing polymer mixture. Poly (ethylene glycol), which has negligible carbon yield upon pyrolysis, was used as a template to study the controlled formation of mesoporosity in pyrolytic carbons. A series of carbons were produced from mixtures of poly (ethylene glycol) and poly (furfuryl alcohol) with $25 \%$ to $75 \%$ composition and 300 to $18500 \mathrm{~g} / \mathrm{mol}$ of template. Polydisperse dextran adsorption reveals a maximum in uptake for 8000 $\mathrm{g} / \mathrm{mol}$ and $50 \%$ templated carbons while materials from $75 \%$ mixtures or those from less than $2000 \mathrm{~g} / \mathrm{mol}$ template yielded negligible dextran uptake. These results correlated well with the prominence of a broad peak between $5^{\circ}$ to $20^{\circ} 2 \theta$ in the X-ray diffraction spectrum and qualitatively with micrographs of fracture surfaces of the carbons. The results suggest a templating process dominated by both the molecular size of the template and the rate of expulsion of decomposed template material during the formation of the solid. At molecular weights below $2000 \mathrm{~g} / \mathrm{mol}$ of PEG, pores are essentially unconnected as the system exists below the percolation threshold of the medium. This results in little adsorption uptake and evidence of retained decomposition material of the template. At high template compositions and molecular weights, removal of template decomposition products is also inhibited resulting in similar structural and adsorptive properties.

\section{Cesium and Nanoporous Carbon Catalysis}

Cesium in nanoporous carbon $(\sim 10-15 \mathrm{wt} \%)$ was observed to be an excellent catalyst for the heterogeneous conversion of 1-butene preferentially to cis-2-butene, indicating an anionic mechanism of reaction. The same catalyst was also able to couple benzene to $\mathrm{di}$ - and triphenyl and to produce isobutylbenzene from propene and toluene under mile conditions. The catalyst will also convert acetone primarily into isophorone and lesser amounts of mesityl oxide. It was also observed that when present at higher concentrations $(>30 \mathrm{wt} \%)$, cesium catalyzes the formation of nanocrystalline domains from the amorphous nanoporous carbon. These domains are best described as multiwalled nanotubes, onions, polyhedra and nanographite. There formation is all the more unusual because the NPC is considered to be a non-crystallizing carbon, that is a carbon which converts to graphite only at temperatures approaching $2000^{\circ} \mathrm{C}$. In the . presence of cesium, the nanocrystallization process takes place rapidly at temperatures as low as $350^{\circ} \mathrm{C}$. 


\section{Summary}

My group is now able to synthesize and fully characterize via permeation characteristics and physical measurements the behavior and properties of nanoporous carbon films supported on stainless steel. We have also been able to show that these materials may be converted readily into catalytic membranes or membrane reactors by incorporation of active sites either within the nanoporous carbon medium itself or by contacting the sites intimately with the surface. Thus we are in a unique position to begin to exploit these findings and this science for new coupled catalysis and separation processes which are important for energy related problems.

The cesium metal catalysts are quite interesting in their own right and we feel that taken in combination with transition metals, we may begin to couple reactions such as alkane dehydrogenation to alkenes together with base and free radical reactions leading to carbon-carbon bond formation. The solid state catalytic reaction of cesium with the amorphous carbon to produce nanographitized domains (polyhedra, onions and multiwalled tubes) is unprecedented and may be a true breakthrough in the reactions of carbon materials.

During the course of this work, and primarily between September 1997 and November 2001, total or partial funding was provided by this contract for completion of the Ph.D. theses of Dr. Madhav Acharya, Dr. Mark G. Stevens, Dr. Michael S. Strano and Dr. Mark B. Shiflett. Partial funding was also made available from this contract for early support of the Ph.D. thesis of Mr. Kevin McNamara, who is due to complete his thesis in summer 2002.

The contract led to 17 papers, either published already or accepted for publication, with two more manuscripts to be submitted this Spring 2002. Two patent applications were also filed under auspices of this research.

\section{Publications due and attributed to DOE BES}

1. Acharya, M. and Foley, H. C. "Spray-Coating of Nanoporous Carbon Membranes for Air Separation," J. Membr. Sci., 1999, $161,1$.

2. Shiflett, M.B. and Foley, H.C. "Ultrasonic Deposition of High-Selectivity Nanoporous Carbon Membranes." Science, 1999, 285, 1902.

3. Shiflett, M.B., Pedrick, J.F., McLean, S.R., Subramoney, S., Foley, H.C. "Characterization of Supported Nanoporous Carbon Membranes." Adv. Mat., 2000, $12,21$.

4. Shiflett, M.B. and Foley, H.C. "Reproducible Production of Nanoporous Carbon Membranes." Carbon, 2001, 39, 1421. 
5. Shiflett, M.B. and Foley, H.C. On the Preparation of Supported Nanoporous Carbon Membranes." J. Membr. Sci., 2000, 179, 275.

6. Shiflett, M.B., Foley, H.C., Yokozeki, A. Theoretical Calculation of Polymer Deposition Thickness on a Cylindrical Substrate." AIChE J., 2001, 47, 1648.

\section{Reactive Nanoporous Membranes}

7. Strano, M. S. and Foley, H. C. "Synthesis and Characterization of Catalytic Nanoporous Carbon Membranes (Pt/NPC)." AIChE J., 2001, 47, 1.

8. Strano, M. S., and Foley, H. C. "Synthesis and Characterization of Heteropolyacid Nanoporous Carbon Membranes." Catal. Lett., 2001, 74, 177.

9. Strano, M. S., and Foley, H. C. "Novel Heteropolyacid Nanoporous Carbon Reactive Barriers for Supra-Equilibrium Conversion and In-Situ Component Separation." J. Phys. Chem. Accepted.

\section{Molecular Transport Across Nanoporous Membranes}

10. Strano, M. S. and Foley, H. C. "Deconvolution of Permeance through Nanoporous Membranes." AIChE J., 2000, 46, 3.

11. Strano, M. S. and Foley, H. C. "Temperature and Pressure Dependent Transient Analysis of Single Component Permeation through Tubular Nanoporous Carbon Membranes." Carbon (in press. submitted Jan. 2001)

12. Strano, M. S. and Foley, H. C. "Modeling Ideal Selectivity Variation in Nanoporous Carbon Membranes." Chem. Eng. Sci. (submitted March 2001)

Mesoporous Adsorbent Synthesis by Templating of Pyrolytic Carbons

13. Strano, M. S., Zydney, A., Barth, H., Wooler, G., Agarwal H. and Foley, H. C. "Ultrafiltration Membrane Synthesis by Nanoscale Templating of Porous Carbon." J. Membr. Sci. 2002, 198, 173.

14. Strano, M. S. and Foley, H. C. "Templating of Pyrolytic Carbons: the Effect of Template Molecular Weight and Composition." Carbon (accepted )

Cesium and Nanoporous Carbon Catalysis and Nanocrystallization

15. Stevens, Mark G., Chen, D. and Foley, H. C. "Oxidized Cesium/Nanoporous Carbon Materials: Solid-Base Catalysts with Highly Dispersed Active Sites," J. C. S., Chem. Commun., 1999, 275. 
16. Stevens, M. G., Anderson, M. R. and Foley, H.C. "Side-Chain Alkylation of Toluene with Propene on Caesium/Nanoporous Carbon Catalysts." J. C. S., Chem. Commun., 1999, 413.

17. Stevens, M.G. and Foley, H. C. "Spontaneous Formation of Carbon Nanotubes and Polyhedra from Cesium and Amorphous Carbon." Chem. Phys. Lett. 1998, 292, 352.

\section{Patents}

1. Strano, M.S., Acharya, M. et. al. "Supported carbogenic molecular sieve membrane and method of producing the same." US Patent \#5972079 with extension to catalytic/reactive systems (extension filed Jan. 2000)

2. Strano, M.S., Agarawal, H. and H. C. Foley "Porous carbon membranes for macromolecular separations." Provisional US patent filed Feb. 2001. 